\title{
Down-regulation of G9a triggers DNA damage response and inhibits colorectal cancer cells proliferation
}

This article has been corrected. Correction in: Oncotarget. 2020; 11:302-303. Jie Zhang ${ }^{1}$, Pengxing $\mathrm{He}^{2}$, Yong $\mathrm{Xi}^{1}$, Meiyu Geng ${ }^{1}$, Yi Chen ${ }^{1}$, Jian Ding ${ }^{1}$

\author{
${ }^{1}$ Division of Anti-Tumor Pharmacology, State Key Laboratory of Drug Research, Shanghai Institute of Materia Medica, Chinese \\ Academy of Sciences, Shanghai 201203, China \\ ${ }^{2}$ School of Pharmaceutical Sciences, Zhengzhou University, Zhengzhou 450001, China \\ Correspondence to: \\ Jian Ding, e-mail: jding@simm.ac.cn \\ Yi Chen, e-mail: ychen@simm.ac.cn \\ Keywords: colorectal cancer, G9a, epigenetics, DNA damage response (DDR), SN38/CPT, synergistic effect \\ Received: August 10, $2014 \quad$ Accepted: November 19, $2014 \quad$ Published: January 20, 2015
}

\section{ABSTRACT}

G9a, a histone methyltransferase, is aberrantly expressed in some human tumor types. By comparing 182 paired colorectal cancer and peritumoral tissues, we found that G9a was highly expressed in colorectal cancer (CRC). Overexpression of G9a promoted CRC cells proliferation and colony formation, whereas knockdown of G9a inhibited CRC cells proliferation. Depletion of G9a increased the rate of chromosome aberration, induced DNA double strand breaks and CRC cells senescence. G9a inhibition synergistically increased YH2AX expression induced by topoisomerase I inhibitors and ultimately led to CRC cell death. The findings that down-regulation of G9a triggers DNA damage response and inhibits colorectal cancer cells proliferation may define G9a as potential oncotarget in CRC.

\section{INTRODUCTION}

Colorectal cancer (CRC) is the third most common cancer and the fourth leading cause of cancer deaths worldwide [1, 2]. The World Cancer Research Fund International estimates that nearly 1.4 million new cases of CRC were clinically diagnosed worldwide in 2012, with 102,480 new cases in the United States [3]. Currently, the main treatment for CRC is surgery, radiotherapy, and chemotherapy. However, the 5-year survival rate is less than $40 \%$ in less developed countries [4]. Following chemotherapy, more than $50 \%$ of patients relapse, and this is associated with metastasis [5]. Advanced stage colorectal tumors are notoriously resistant to chemotherapy and show a very poor 5 -year survival $[6,7]$. For these reasons, research into the mechanisms behind CRC development is imperative for the identification of novel therapeutic targets.

CRC has been depicted for many years as a prototypic model for the genetic basis of cancer, and is now becoming increasingly cited as an epigenetic model for tumorigenesis. In fact, epigenetic alterations, including DNA methylation and histone modification, are much more prevalent than genetic alterations in the development of CRC $[4,8,9]$. These epigenetic changes that lead to heritable changes in gene expression are now widely used as diagnostic and prognostic molecular markers, as well as novel therapeutic targets.

G9a, which is also known as EHMT2/KMT1C, is a histone methyltransferase (HMT) that contains a $\mathrm{Su}$ (var), Enhancer of Zeste, Trithorax (SET) domain, and localizes in euchromatin regions where it catalyzes the mono- and di-methylation of histone H3 Lys9 (H3K9me1/ $\mathrm{H} 3 \mathrm{~K} 9 \mathrm{me} 2)$ [10]. In addition to histone modifications, some non-histone G9a substrates such as p53 [11] have been identified. Evidence suggests that G9a is required for the maintenance of the malignant phenotype. Dysregulated expression of G9a was documented in different tumor types, including lung cancer, breast cancer, and hepatocellular carcinoma [12-14]. However, the tumorigenic role of G9a in colon cancer is far from clear. In the present study, we aimed to explore whether G9a plays an important role in the development of CRC.

\section{RESULTS}

\section{G9a is highly expressed in CRC patient samples}

To elucidate a link between G9a expression and $\mathrm{CRC}$, immunohistochemical staining was used to analyze G9a levels in normal and tumor tissues. In all CRC patient 
tissues, G9a staining was observed mainly in the cell nucleus, with cytoplasmic staining in few specimens. Examination of 182 paired CRC specimens revealed a significantly higher expression of G9a in tumor tissues $(P<0.0001$; Figure 1A, 1B). We also used a panel of $\mathrm{CRC}$ cell lines to profile the expression pattern of G9a. Western blot analysis showed that G9a was expressed in all CRC cell lines tested (Figure 1C). Our data collectively demonstrated that G9a is highly expressed in both clinical samples and CRC cell lines, suggesting a potential role of $\mathrm{G} 9 \mathrm{a}$ in maintaining the malignant phenotype of CRC.

\section{G9a is important for colon cancer cell proliferation in vitro and in vivo}

In order to explore the role of G9a in colorectal cancer development, specific siRNAs were used to knockdown G9a expression in HT29, SW620, KM12, SW480, and HCT15 CRC cell lines. siRNA transfection successfully reduced G9a expression, and consequently suppressed $\mathrm{H} 3 \mathrm{~K} 9 \mathrm{me} 2$ in all CRC cell lines, whereas no difference in GLP expression was observed. The knockdown of G9a drastically reduced cell growth in vitro (Figure 2A). To further assess the effects of G9a expression on cell growth, stable cell lines were generated with limited G9a expression (shG9a1, shG9a2, shG9a3 in HT29, and shG9a1, shG9a2 in SW620) (Figure 2B) and abundant G9a expression (pLEX-hG9a transfected in HT29 and SW620) (Figure 3A). As compared with the parent cells, the cells that stably suppressed G9a expression grew more slowly (Figure 2B), and possessed a reduced capacity for colony formation (Figure 2C). In contrast, overexpression of G9a promoted CRC growth in vitro (Figure $3 \mathrm{~A}, 3 \mathrm{~B}$ ). To further substantiate these observations, the G9a specific inhibitors, UNC0638 and BIX01294 were used. These inhibitors significantly reduced CRC cell proliferation, with the $\mathrm{IC}_{50}$ values ranging from 1-20 $\mu \mathrm{M}$ (Figure 2D). Our data together suggest that G9a plays a critical role in CRC cell proliferation.

To assess the effect of G9a expression on cell proliferation in vivo, CRC cells with different levels of G9a were subcutaneously inoculated in nude mice. All mice developed palpable cancers within 30 days after inoculation, however silencing G9a impaired tumor growth. As shown in Figure 2E, knockdown of G9a expression with shG9a2 most proficiently attenuated HT29 cell growth in nude mice in comparison to the shCon, shG9a1, and shG9a3 groups, with tumor volumes of $266 \pm 102 \mathrm{~mm}^{3}, 1678 \pm 593 \mathrm{~mm}^{3}, 701 \pm$ $331 \mathrm{~mm}^{3}$, and $930 \pm 194 \mathrm{~mm}^{3}$, respectively on the $32 \mathrm{nd}$ day. Alternatively, the tumor volume in the HT29pLEXhG9a group was statistically larger than that in HT29-pLEXmock, with the tumor volume of the former being $1578 \pm 100 \mathrm{~mm}^{3}$, while the latter tumor volume was $978 \pm 132 \mathrm{~mm}^{3}$ on the $21 \mathrm{st}$ day (Figure 3C). All these strongly suggest that G9a can regulate the tumor growth of CRC.

\section{Down-regulation of G9a induces DNA damage response in colon cancer}

It has been reported that down-regulation of G9a can induce chromosome instability in cancer cells [15]. By means of karyotype analysis, we found that knockdown of G9a increased the rate of chromosome aberration from $0.55 \%$ to $5 \%$ in HT29shG9a cells, as

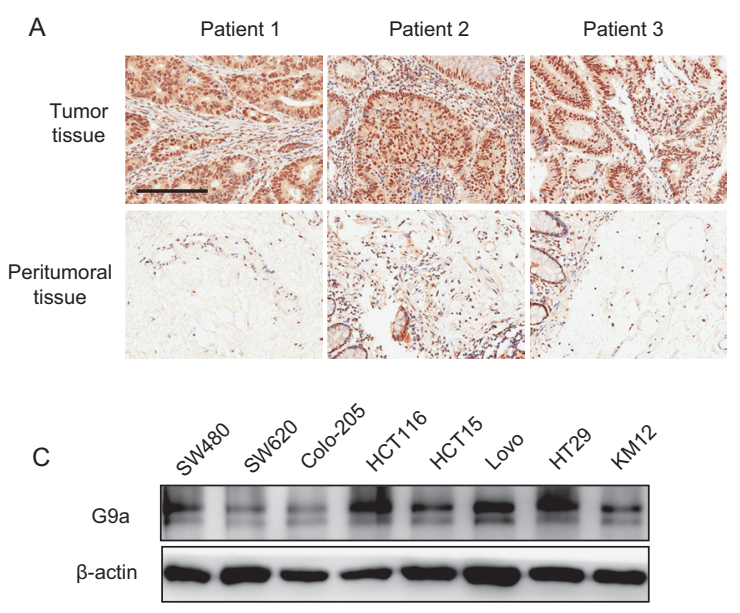

B

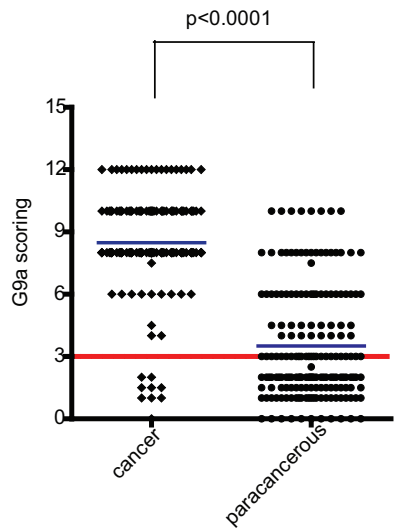

Figure 1: G9a is highly expressed in colorectal cancer. (A) Representative photomicrographs of G9a immunohistochemical staining from CRC tumors and peritumoral tissues (scale bar 1mm). (B) G9a immunohistochemical staining scores for each specimen. Each symbol represents a tumor from an individual patient while the blue lines are group mean scores. The red line is dividing the high and low expression levels of G9a based on the staining scores of immunohistochemical in CRC patients. (C) Protein levels of G9a in human $\mathrm{CRC}$ cell lines as determined by western blot analyses. 
A
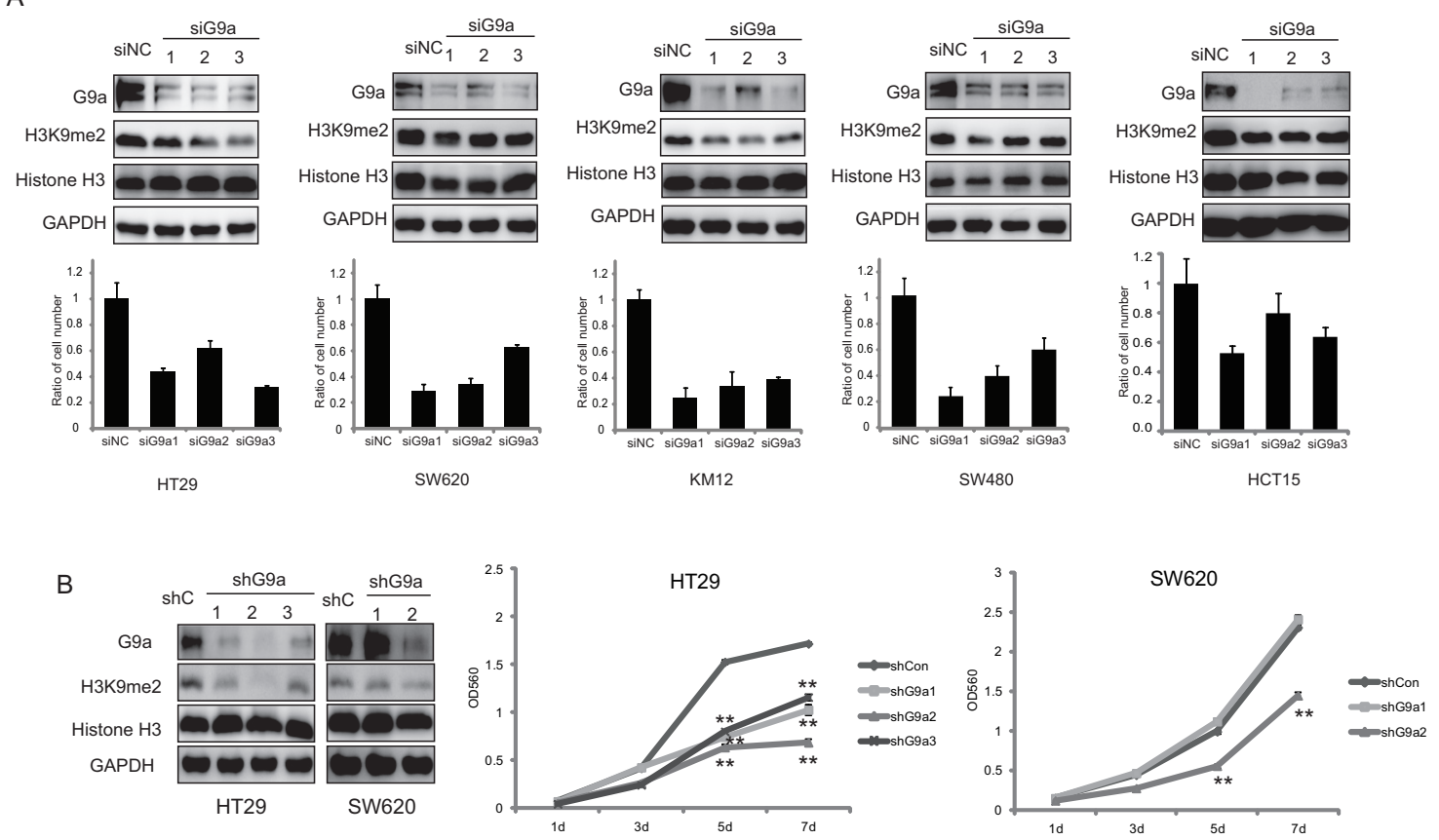

C
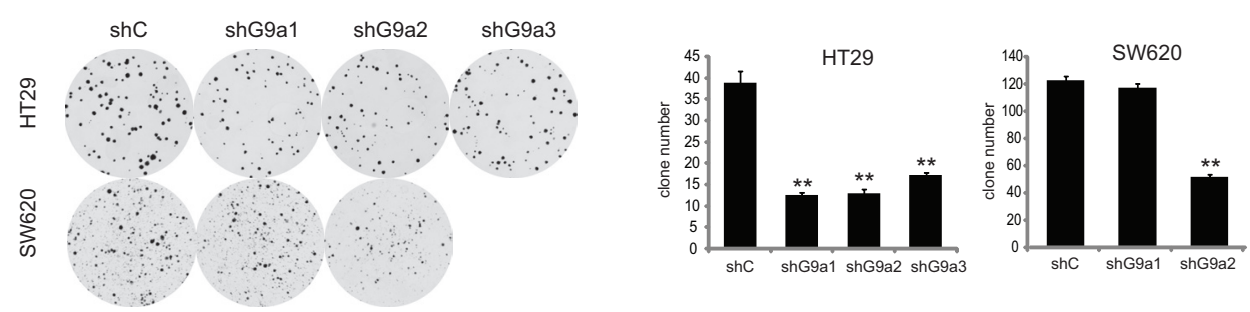

D

UNC0638

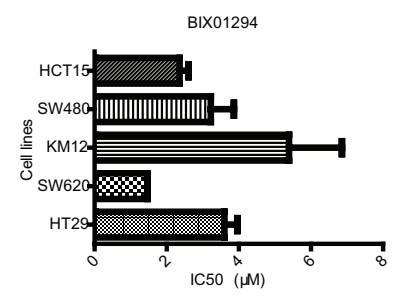

$\mathrm{E}$
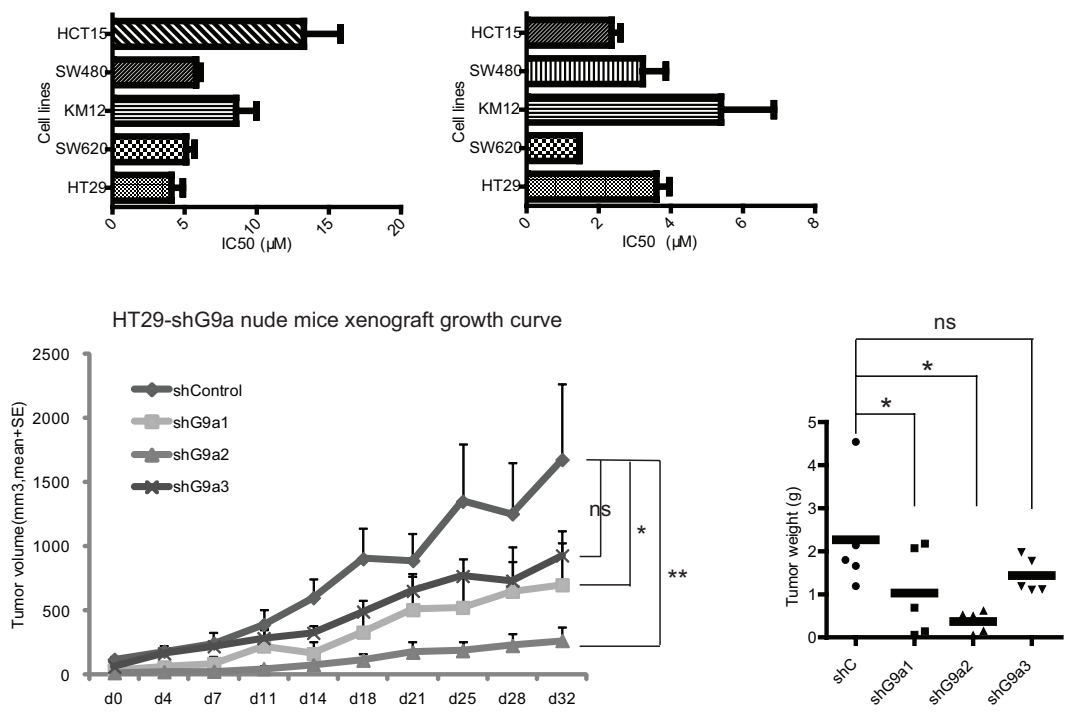

Figure 2: G9a is important to CRC cell proliferation in vitro and in vivo. (A) Different siRNAs for G9a reduced G9a expression and delayed CRC cell growth in vitro. Histograms show the relative cell proliferation at $120 \mathrm{~h}$ after siRNA transfection. Delayed cell growth (B) and reduced colony formation (C) for HT29 and SW620 cells stably suppressing G9a expression. (D) CRC cells treated with different concentrations of the G9a inhibitors UNC0638 and BIX01294. Inhibitory effects of these inhibitors on CRC cell proliferation were measured by the SRB assay (mean $\mathrm{IC}_{50}$ values were calculated from at least three independent experiments). (E) HT29 cell growth following knockdown of G9a expression and inoculation of cells in nude mice. Data are expressed as the mean $\pm \mathrm{SE}$. Tumor weight is also shown. $* * P<0.01, * P<0.05$. 
A

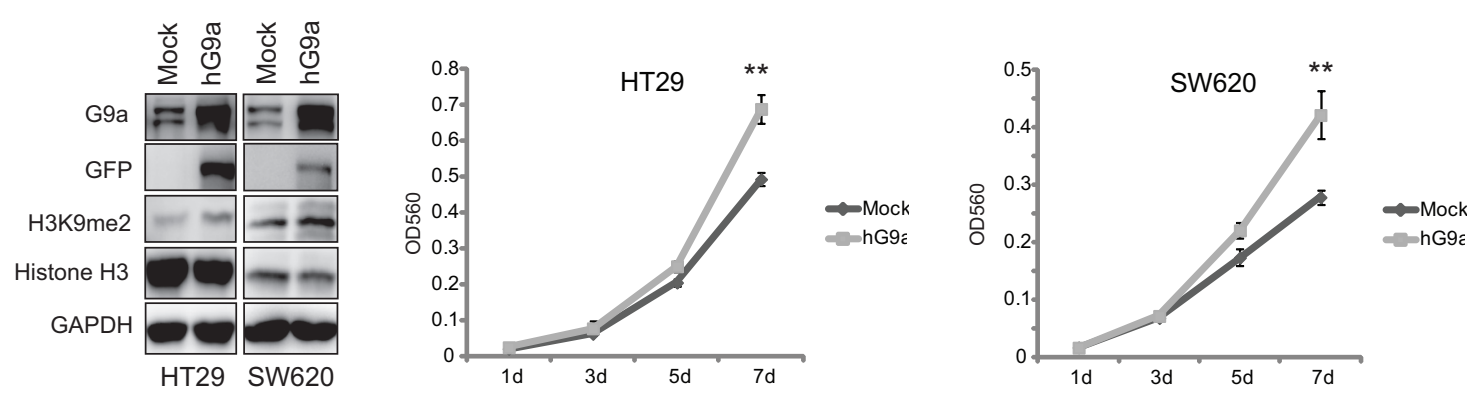

B

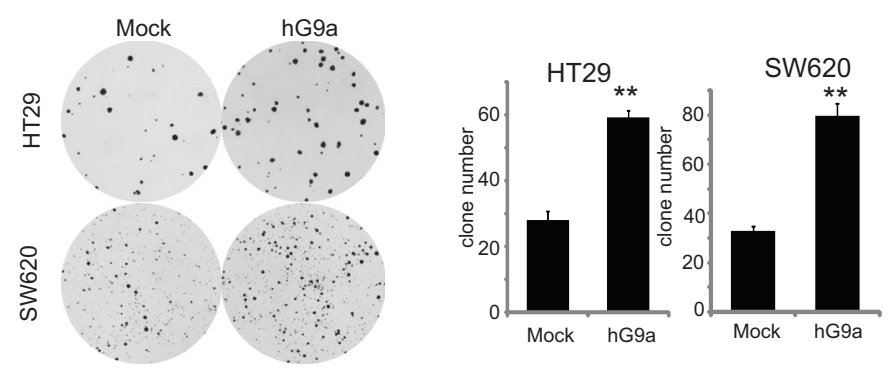

C
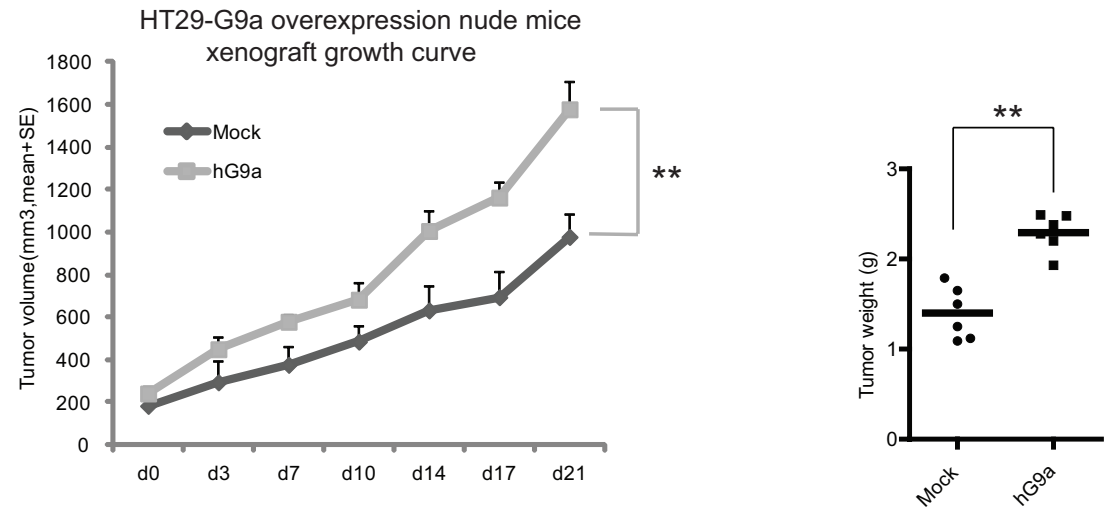

Figure 3: Up-regulation of G9a enhances colon cancer cell proliferation. Overexpressed G9a in HT29 and SW620 promotes (A) cell growth and (B) colony formation in vitro. Bars indicate standard deviation of the mean for $n>3$ determinations. (C) G9a overexpression increased the growth of the HT29 cells inoculated in nude mice. Data are expressed as the mean $\pm \mathrm{SE}$. Tumor weight is also shown. ${ }^{* *} P<0.01$.

compared with cells transfected with shCon (Figure 4A). Given that chromosome instability leads to DNA damage [16], we used a neutral comet assay, a simple, sensitive and rapid method for the detection and quantification of DNA damage [17], to evaluate whether G9a depletion induces DNA double-strand breaks (DSBs). In Figure 4B, the degree of cellular DNA DSBs increased after G9a knockdown in HT29 and SW620 cells, as evidenced by the frequent appearance and expanding volume of comet tails, as well as the shrinkage of comet heads. Furthermore, we found an increased expression of phosphorylated H2AX $(\gamma \mathrm{H} 2 \mathrm{AX})$, which is a well-known marker of DNA DSBs.

Since $\gamma \mathrm{H} 2 \mathrm{AX}$ is known to be phosphorylated by members of phosphoinositide 3-kinase related protein kinases (PIKKs) such as ATM (ataxia-telangiectasia, mutated), ATR (ATM and Rad-related kinase), or DNAdependent protein kinase catalytic subunit (DNA-PKcs) in response to genomic insult [18], we further investigated the potential effect of G9a on these upstream signaling molecules. We found that levels of p-ATM (Ser 1981), p-ATR (Ser 428), ATM, p-Chk1 (Ser 317), and p-Chk2 (Thr 68) increased in G9a-knockdown HT29 and SW620 cell lines as compared to cells transfected with shCon (Figure 4C, Figure S1). Similar results were observed in the in vivo studies. We found that Ki67, a hallmark of proliferation, decreased in G9a-knockdown HT29 xenografts, followed by an increased level of $\gamma \mathrm{H} 2 \mathrm{AX}$ (Figure 4D, 4E). These studies indicate that suppression of G9a expression triggers DSBs and a robust DNA-damage response in colon cancer. 
A
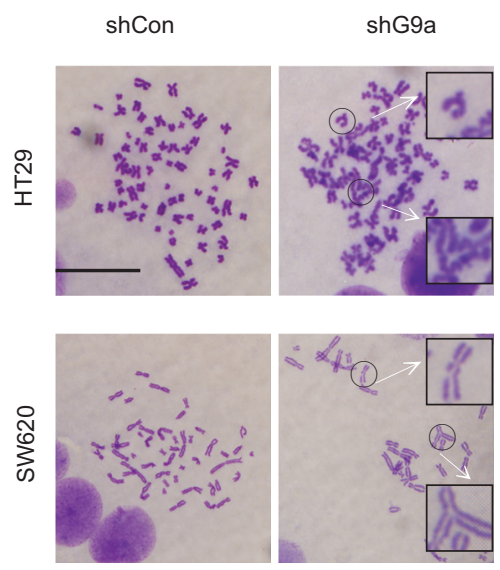
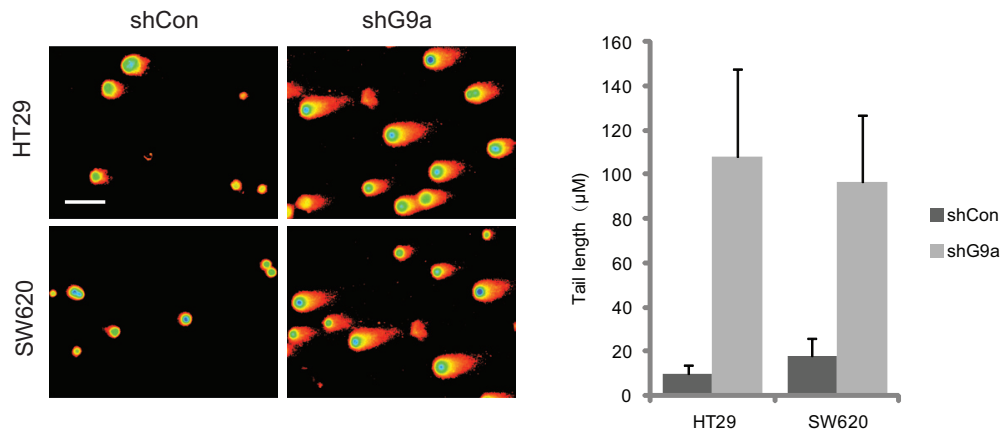

C

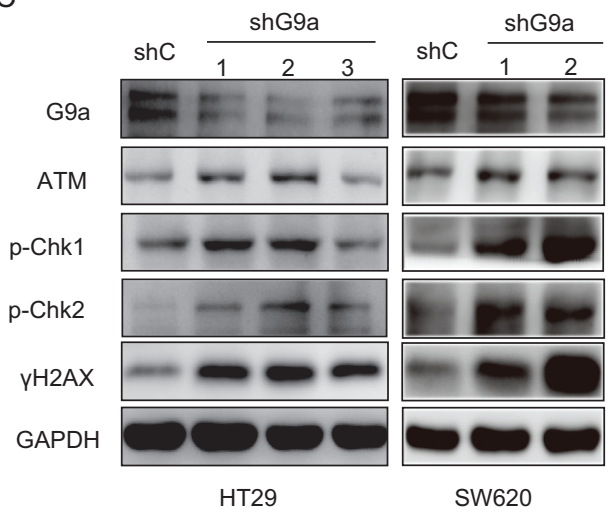

D

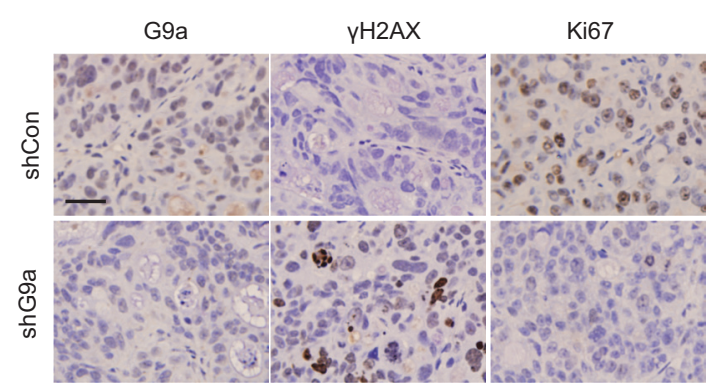

$\mathrm{E}$
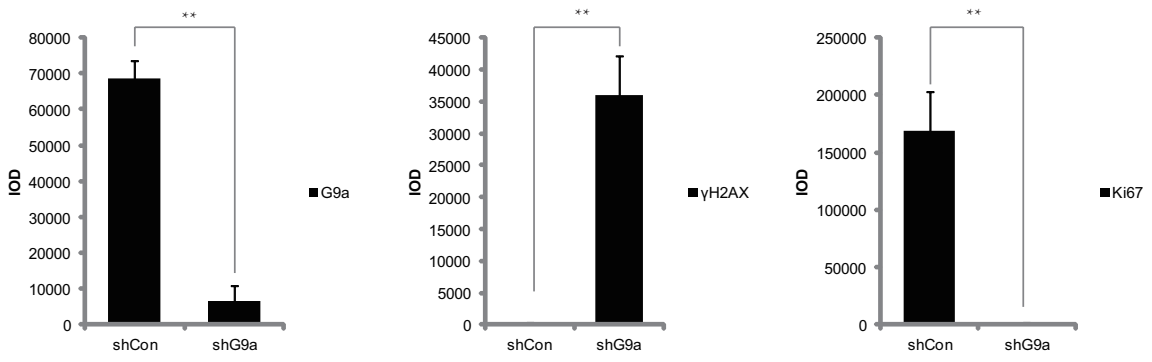

Figure 4: Down-regulation of G9a induces DNA damage in colon cancer. (A) Karyotype analysis showed that down-regulation of G9a induced chromosome instability (scale bar $10 \mu \mathrm{M}$ ). (B) DNA damage as assessed by the comet assay (scale bar $25 \mu \mathrm{M})$. (C) The protein expression levels for G9a, ATM, p-Chk1 (Ser 317), p-Chk2 (Thr 68), $\gamma \mathrm{H} 2 \mathrm{AX}$, and GAPDH (loading control) following the stable knockdown of G9a in HT29 and SW620 cells. (D) Representative immunohistochemical staining images of G9a, Ki67, and $\gamma \mathrm{H} 2 \mathrm{AX}$ in xenografts derived from HT29 cells that stably silenced G9a expression (scale bar $25 \mu \mathrm{M}$ ). (E) The quantified results by Image Pro Plus based on the immunohistochemical staining in Figure 4D. ${ }^{*} P<0.01$.

\section{Silencing G9a leads to cancer cell senescence}

DNA damage often leads to a halt in cell proliferation by triggering apoptosis or senescence, which thereby prevents transmission of harmful mutations onto daughter cells $[19,20]$. And $\gamma-\mathrm{H} 2 \mathrm{AX}$ is not only a marker of DNA damage but also a marker of cellular senescence even in the absence of DNA damage
$[21,22]$. In this regard, we investigated whether G9a knockdown may induce senescence of CRC cells. Since SA- $\beta$-gal (senescence-associated $\beta$-galactosidase) has been identified as a specific marker for senescent cells [23], HT29 and SW620 cells were then stained with $\beta$-galactosidase to determine whether senescence was initiated by G9a depletion-induced DNA damage response (DDR). We found a significant increase of $\beta$-galactosidase 
positive cells when G9a was suppressed in the CRC cell lines (Figure 5A). Moreover, p21, which is considered an inducer of cellular senescence, was up-regulated in these cell lines (Figure 5B). These findings were consistent with those observed in HT29 xenograft tumors that had G9a stably suppressed (Figure 5C). However, knockdown of G9a failed to induce apoptosis, as evidenced by the consistent annexin V+ levels in HT29shG9a cells and SW620shG9a cells (Figure 5D), when compared with shCon cells.

\section{G9a depletion synergizes with topoisomerase I (TOPO I) inhibitors in CRC cells}

Combinatorial drug treatment is widely used in cancer therapeutics to combat single-drug resistance, overcome side effects, and increase sensitivity to therapy. Since TOPO I inhibitors are widely used to treat CRC, we investigated whether G9a would potentiate the anticancer activity induced by TOPO I inhibitors. For this, we used two TOPO I inhibitors SN-38 and CPT as combinatorial components. We found that the $\mathrm{IC}_{50} \mathrm{~s}$ for $\mathrm{SN}-38$ and CPT were lower in G9a knockdown cells, as compared with parent cells (Figure 6A). We next determined the combination therapy effect by calculating the combination index (CI) using the CalcuSyn program, which is based on the Chou-Talalay method [24]. A CI $<1$ indicates a synergistic effect, $\mathrm{CI}=1$ indicates an additive effect, whereas $\mathrm{CI}>1$ indicates an antagonistic effect. Our data shows that the TOPO I inhibitors and the G9a inhibitor UNC0638 displayed a synergistic effect in a panel of CRC cell lines, including HT29, SW620, HCT-15, Colo-205, KM12, and LoVo (Figure 6B, 6C). Western blot and immunofluorescence analysis further revealed that either UN0638 or TOPO I inhibitors alone weakly increased the accumulation of $\gamma \mathrm{H} 2 \mathrm{AX}$ in vitro. However, the combined therapy resulted in a dramatic increase in $\gamma \mathrm{H} 2 \mathrm{AX}$ accumulation (Figure 6D, 6E). Consistent with this, increased phosphorylation of p-Chk1 and p-Chk2 was also observed (Figure 6E).

In addition to the above experiments, the $293 \mathrm{~T}$ cell line was used to generate three stable cell lines: mock (pLEX-Mock), high expression of G9a (pLEX-hG9a), and non-activated mutant-dead G9a (pLEX- $\triangle$ SET-hG9a) (Figure S2). We found that p-Chk1 (Ser 317), p-Chk2 (Thr 68), and $\gamma$-H2AX levels quickly increased in mock and pLEX- $\triangle$ SET-hG9a $293 \mathrm{~T}$ cells in response to CPT treatment. However, no alteration in p-Chk1 (Ser 317), p-Chk2 (Thr 68), and $\gamma$-H2AX were observed with CPT treatment of the pLEX-hG9a cell line (Figure 6F). This suggests that G9a depletion-synergized DNA damage depends on its catalytic activity. Taken together, these
A

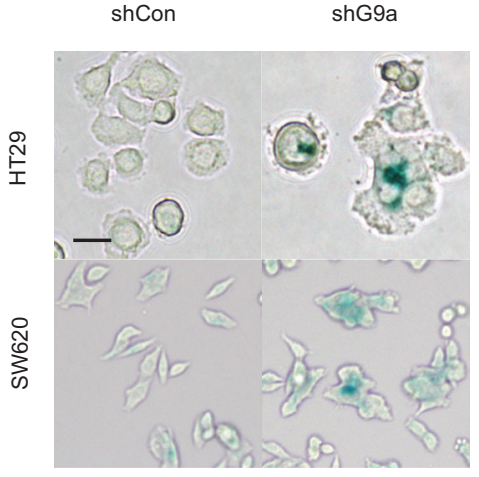

C

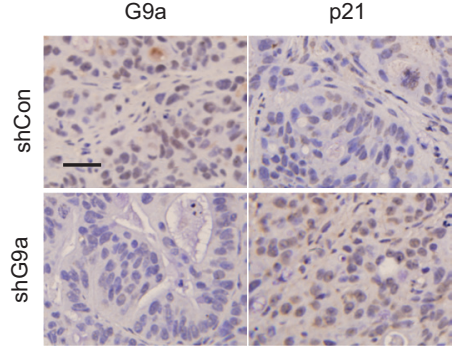

B

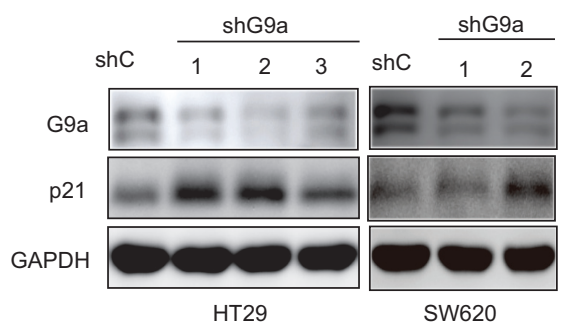

D

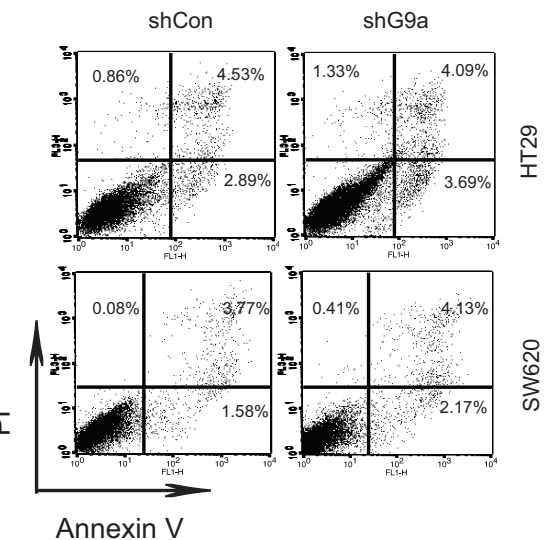

Figure 5: Depletion of G9a results in senescence. (A) Senescence-associated $\beta$-galactosidase expression in HT29 and SW620 cells following stable knockdown of G9a (scale bar $25 \mu \mathrm{M}$ ). (B) The p21 protein level detected in HT29 and SW620 cells in vitro following the stable suppression of G9a. (C) The p21 protein level detected in HT29 shG9a xenografts (scale bar $25 \mu$ M). (D) Silencing of G9a can not induce CRC cell apoptosis. Percentage of cells in apoptosis was measured by flow cytometry using annexin V and PI staining. The panel in the lower right (annexin $\mathrm{V}^{+} / \mathrm{PI}^{-}$) was considered as cells in early apoptosis. Each condition was studied at $n \geq 3$. ${ }^{*} P<0.05$. 

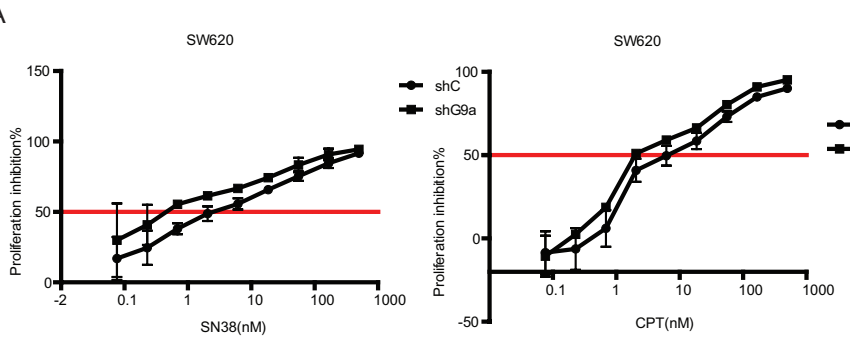

B

C
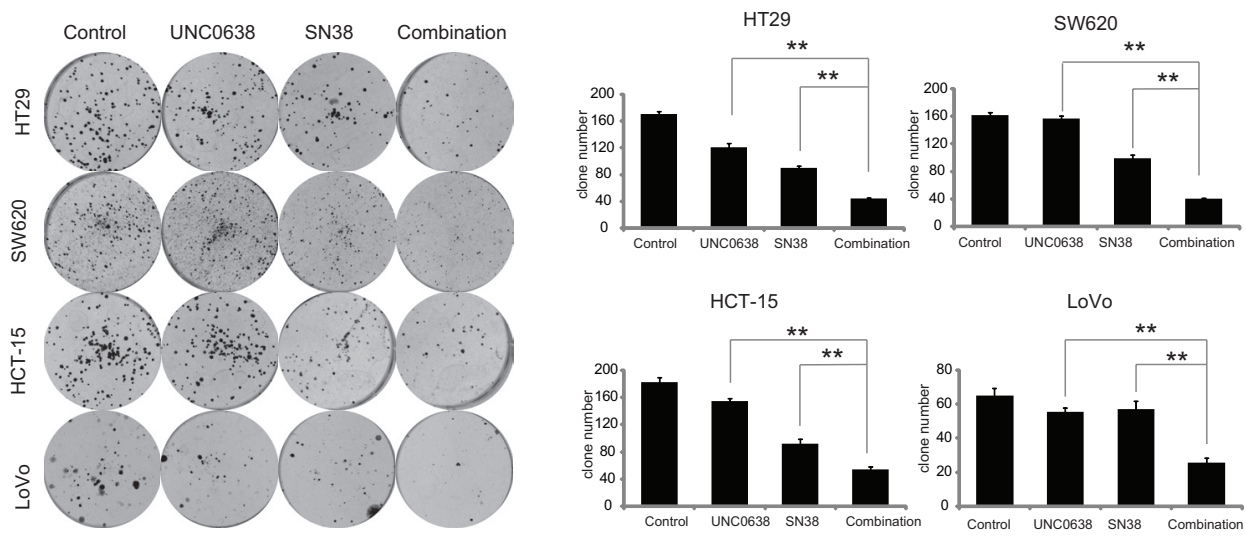

D
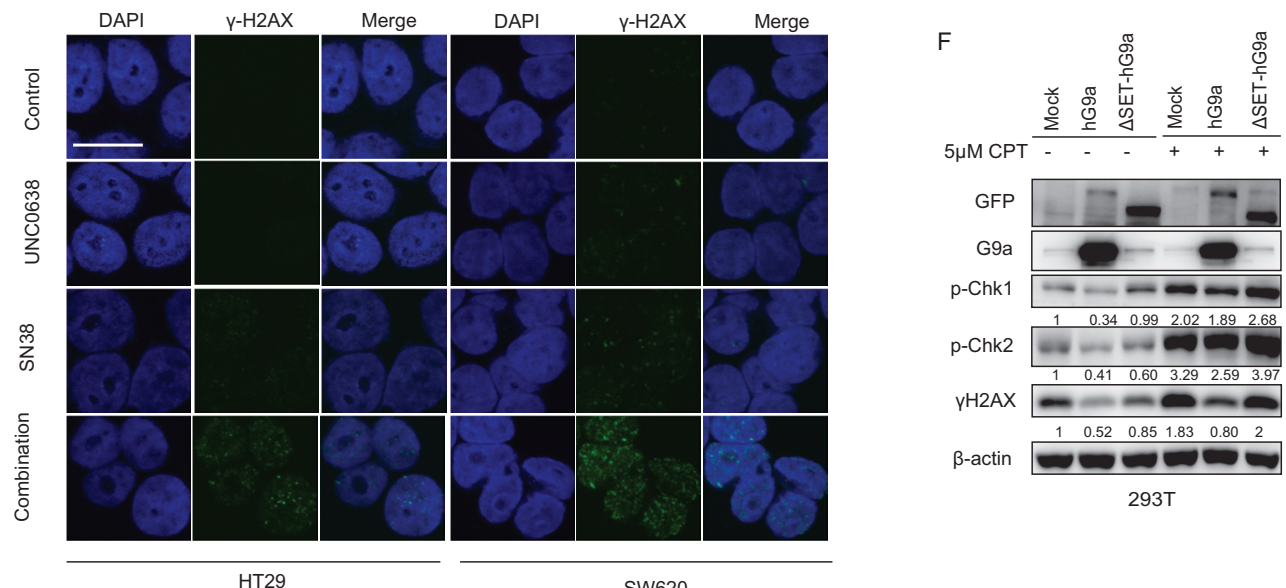

HT29

E

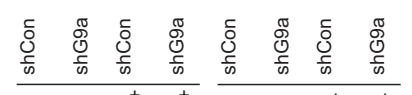

SN38

G9a

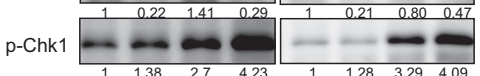

p-Chk2

YH2AX

$\beta$-actin
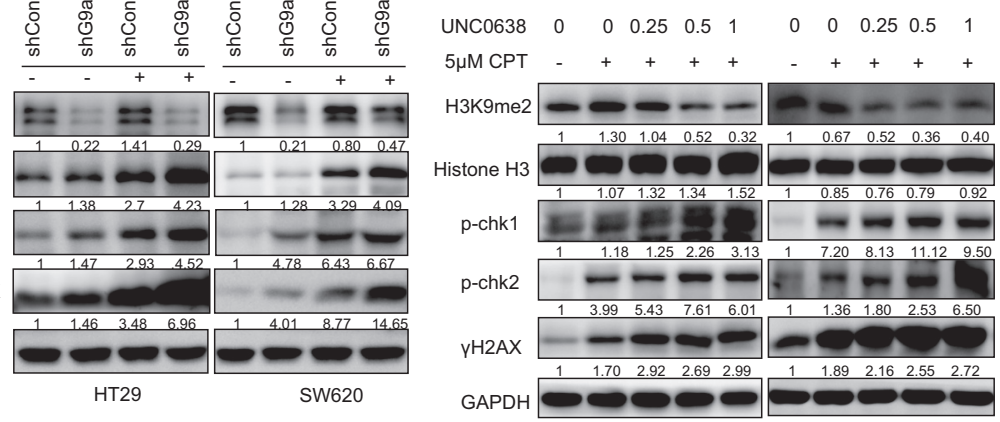

HT29

SW620

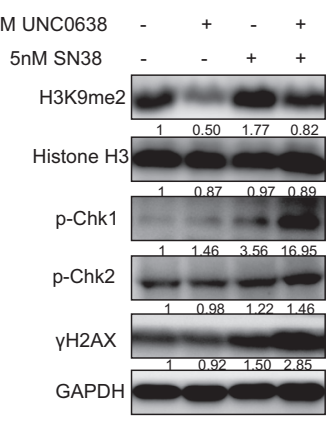

HT29

Figure 6: G9a depletion synergizes with TOPO I inhibitors in CRC cells. (A) SW620 shG9a or shCon cells were treated with different concentrations of SN38 or CPT, followed by subsequent proliferation inhibition assessment using the SRB assay. CRC cells were treated with different concentrations of UNC0638 and/or SN38, and (B) cell proliferation was measured with the SRB assay and CIs were calculated, (C) colony formation was measured. (D) Protein levels of $\gamma \mathrm{H} 2 \mathrm{AX}$ were analyzed by microscopy (scale bar $10 \mu \mathrm{M})$, and (E) protein levels of histone H3, H3K9me2, p-Chk1, p-Chk2, $\gamma \mathrm{H} 2 \mathrm{AX}$ and GAPDH were determined by western blot. (F) Protein levels of GFP, G9a, p-Chk1(Ser 317), p-Chk2 (Thr 68), $\gamma \mathrm{H} 2 \mathrm{AX}$, and $\beta$-actin (loading control) from 293T cells stably transfected with pLEX-mock, pLEX-hG9a, or pLEX- $\triangle$ SET-hG9a and treated with the indicated concentrations of CPT. Data are means \pm SD, $n \geq 3 . * * P<0.01$. 
results indicate that G9a depletion synergizes with the antitumor activity of topoisomerase inhibitors in colon cancer treatment.

\section{DISCUSSION}

In the present study, we identified for the first time that over-expression of G9a stimulated CRC cell proliferation both in vitro and in vivo. Moreover, G9aspecific siRNAs, G9a stable knockdown, and G9a specific inhibitors inhibit CRC cell proliferation. Mechanistically, the tumor-promoting effect of G9a appears to bypass its known downstream signaling molecules such as EpCAM [25], PPAR $\gamma$ [26], and Wnt [26, 27] (Figure S3). Instead, G9a depletion increased the rate of chromosome aberration, induced DNA DSBs, and ultimately led to $\mathrm{CRC}$ tumor growth arrest. A remarkable increase in $\gamma \mathrm{H} 2 \mathrm{AX}$ levels in G9a-knockdown CRC cells was also displayed, followed by the accumulation of p-ATM and p-ATR, which is preferentially activated by DSBs. HMTs' abnormality associated with DDR has been reported these years $[28,29]$. Dot1L, which is the only non-SET domain-containing HMT that targets histone H3 lysine 79 (H3K79), can recruit DNA repair component 53BP1 to the sites of damage [30, 31]. EZH2 depletion results in the abrogation of cell cycle G1 and G2/M checkpoints, directing DNA damage response toward predominant apoptosis in both p53-proficient and p53-deficient cancer cells, but not in normal cells [32]. Study of G9a knockout mice showed that G9a was necessary for embryonic development and cell differentiation. It is believed that this embryonic growth defect in G9a-deficient embryonic stem cells may be due to apoptotic cell death but not cell cycle arrest. In that model, chromosomal instability was not observed in the knockout cells [33]. However, it has been reported that an increased number of chromosomes was found in the G9a-knockdown MCF-7 and H1299 cells [13]. The present study provided insight into the possible benefits of down-regulating G9a in colon cancer, with G9a knockdown inducing extensive chromosome instability, which could trigger DNA damage response, cellular senescence, and ultimately tumor growth arrest.

Tumor recurrence following surgery and adjuvant treatment remains a major problem in CRC treatment. Irinotecan (CPT-11) is often used as a first- and secondline chemotherapy for advanced or recurrent CRC. However, only $20-30 \%$ of patients show an objective response to CPT-11. There is increasing awareness of an improved outcome in clinical practice when epigenetic inhibitors and cytotoxic agents are combined [34]. Therefore, we also tried to evaluate a combination therapy of G9a inhibitor and TOPO I inhibitors in CRC treatment. As expected, a synergistic anticancer effect of UNC0638 with TOPO I inhibitors were observed in CRC cells. CI values and the significant decline in the colony numbers when the drugs were combined strongly demonstrated that
G9a depletion potentiated the SN-38-induced cytotoxicity in CRC cells. Furthermore, the combined therapy synergistically increased $\gamma \mathrm{H} 2 \mathrm{AX}$, caused DNA damage, and led to CRC cell senescence.

In summary, the results obtained in this study indicate that G9a inhibition may be a promising therapeutic target in the treatment of CRC, especially when G9a inhibitors are combined with other cytotoxic agents that have a synergistic effect.

\section{MATERIALS AND METHODS}

\section{Chemicals and antibodies}

UNC0638, CPT were both purchased from Sigma (St. Louis, MO, USA). Senescence $\beta$-Galactosidase Staining Kit, RIPA were purchased from Beyotime (Nantong, China). The following antibodies were used as primary antibodies: G9a (Cell Signaling, Danvers, MA, USA), ATM (Cell Signaling), p-Chk1 (Ser 317), p-Chk2 (Thr 68), $\gamma$ H2AX (Santa Cruz Biotechnology, Santa Cruz, CA, USA), Histone H3 (Cell Signaling), Histone H3 Dimethyl (K9) antibody (Epitomics, CA, USA), EpCAM (Epitomics), PPAR $\gamma$ (Cell signaling), Wnt6 (Epitomics), Wnt10a (Epitomics), $\beta$-actin (Cell Signaling), GAPDH (Cell Signaling), GFP (Santa Cruz Biotechnology), Alexa Fluor 488 Goat Anti-Mouse IgG (H+L) Antibody (Life Technologies, Oregon, USA), Alexa Fluor 594 Goat AntiRabbit IgG (H+L) Antibody (Life Technologies).

\section{Immunohistochemistry}

CRC tissue was provided by Shanghai Biochip Company Ltd. The sections consisted of 182 pairs of tumor and matched peritumoral samples. Immunohistochemistry was performed as described previously [35]. Briefly, the EnVision ${ }^{\mathrm{TM}}$ Detection Systems Peroxidase/DAB detection kit was used in this experiment. The primary antibody was diluted at 1:1000 and incubated at $4{ }^{\circ} \mathrm{C}$ overnight. The slides were counterstained with hematoxylin and the tissue image was captured with a digital camera and quantitated by Image Pro Plus.

\section{Cell culture}

The human colon adenocarcinoma HT29, SW620, SW480, HCT15, LoVo, 293T, and 293FT cell lines were purchased from American Type Culture Collection (Manassas, VA, USA). KM12 was from Japan and Colo205 was from Shanghai Institute of Biochemistry and Cell Biology. HT29 and HCT116 cells were grown in McCoy's 5A medium (Sigma) supplemented with 10\% fetal bovine serum (FBS; Gibco, Grand Island, NY). SW620 and SW480 cells were grown in L-15 (Gibco, Grand Island, NY) supplemented with 10\% FBS. HCT15 and Colo-205 were grown in RPMI-1640 (Gibco, Grand 
Island, NY) supplemented with $4.5 \mathrm{~g} / \mathrm{L}$ glucose, $0.11 \mathrm{~g} / \mathrm{L}$ sodium pyruvate, and $10 \%$ FBS. LoVo was grown in F-12 (Gibco, Grand Island, NY) supplemented with $0.29 \mathrm{~g} / \mathrm{L}$ glutamine and 10\% FBS. KM12 was grown in RPMI-1640 (Gibco, Grand Island, NY) supplemented with 10\% FBS. The 293FT Lentiviral Expression System and 293T were cultured in Delbecco's modified Eagle medium (Gibco, Grand Island, NY) supplemented with 10\% FBS.

\section{Plasmids and transfection}

pLKO.1-shG9a plasmids were generous gifts from Dr. Jin Jian (University of North Carolina, USA). pEGFPhG9a, pEGFP- $\triangle$ SET-hG9a [36] were obtained from Addgene, and recombined into pLEX to construct pLEXhG9a, and pLEX- $\triangle$ SET-hG9a. These plasmids were transfected into 293FT cells with packaging mix (pCMV$\mathrm{dR} 8.2 \mathrm{dvpr}$ and pCMV-VSVG) to produce lentiviruses. The stable knockdown and overexpressed G9a cell lines were established as outlined in the Addgene protocols.

\section{Quantitative real time RT-PCR}

Total cellular RNA was isolated with TRIzol (Invitrogen, Carlsbad, CA, USA) and reverse transcribed into cDNA using the PrimeScript ${ }^{\mathrm{TM}}$ RT reagent Kit (Takara, Otsu, Shiga, Japan). Real-time RT-PCR was performed on an Applied Biosystems 7500 using SYBR-Green Master mix (Takara) with the following primers: G9a 5'- gccaggecgggaggcectggaa-3' (sense), 5'- ctccagcctgcagcagcacatg-3' (antisense); GLP 5'- gccgtggacagcgagccatgcccc-3' (sense), 5'ggcaggagccggccatccttgtcgt-3' (antisense), and GAPDH 5'gcaaattccatggcaccgtc- $3^{\prime}$ (sense), 5'- tcgccccacttgattttg- $3^{\prime}$ (antisense). The reactions parameters were: $95^{\circ} \mathrm{C}$ for $10 \mathrm{~min}$ followed by 42 cycles of $95^{\circ} \mathrm{C}$ for $5 \mathrm{~s}$ and $60^{\circ} \mathrm{C}$ for $34 \mathrm{~s}$. All samples including the template controls were assayed in triplicate. The relative number of target transcripts was normalized to the number of human GAPDH transcripts found in the same sample. The relative quantification of target gene expression was performed with the standard curve or comparative cycle threshold (CT) method.

\section{Colony formation assay}

SW620 and HT29 stable knockdown or overexpressed cell lines were seeded in 6-well plates at the density of 200 cells per well. The cells were cultured for 10-15 days until colonies were visible. Colonies were fixed in $10 \%$ formaldehyde and $10 \%$ acetic acid at room temperature for 10 min and then stained with $1 \%$ crystal violet.

\section{Sulforhodamine B (SRB) assay}

Cells were seeded onto 96-well plates with the same density, and cultured for $1 \mathrm{~d}, 3 \mathrm{~d}, 5 \mathrm{~d}$, and $7 \mathrm{~d}$. Cells were then fixed with $10 \%$ trichloroacetic acid (TCA), washed with distilled water, and stained with SRB (Sigma, St. Louis, MO, USA) in 1\% acetic acid. SRB in the cells was dissolved in $10 \mathrm{mM}$ Tris- $\mathrm{HCl}$ and was measured at $560 \mathrm{~nm}$ with a spectraMAX190 (Molecular Devices, Sunnyvale, CA).

\section{Western blot analyses}

Cells were collected and lysed in RIPA (Beyotime, Nan-tong, China) supplemented with a protease inhibitor cocktail (Roche, Mannheim, Germany). An equal amount of protein was subjected to SDS-PAGE and transferred to nitrocellulose membranes, and probed with primary antibodies at $4^{\circ} \mathrm{C}$ overnight. Protein levels were quantified using Image $\mathrm{J}$.

\section{Comet assay [35]}

Slides were pre-coated with $1 \%$ normal melting point agarose. About $3 \times 10^{4}$ cells were mixed with $70 \mu \mathrm{L}$ of $1 \%$ low melting point agarose in phosphate-buffered saline (PBS), and rapidly spread onto the pre-coated slides. The slides were immediately placed in cold lysis buffer containing $2.5 \mathrm{M} \mathrm{NaCl}, 100 \mathrm{mM}$ EDTA, $10 \mathrm{mM}$ Tris (pH 7.5), $1 \%$ Triton X-100, $1 \%$ INCI, and $10 \%$ DMSO at $4{ }^{\circ} \mathrm{C}$ for $1-3 \mathrm{~h}$. The slides were then placed in the electrophoresis solution for $20 \mathrm{~min}$ to facilitate DNA unwinding before electrophoresis was conducted for 20 $\mathrm{min}$ at $25 \mathrm{~V}$ and $300 \mathrm{~mA}$. After electrophoresis, the slides were washed with PBS and then stained with DAPI. The individual cells were viewed using an Olympus BX51 UV fluorescence microscope(Olympus, Japan).

\section{Karyotype analysis}

Colcemid (final concentration $0.1 \mu \mathrm{g} / \mathrm{mL}$ ) was added to culture flasks and incubated at $37^{\circ} \mathrm{C}$ for $4 \mathrm{~h}$, before cells were trypsinized, collected and centrifuged at 1000 $\mathrm{rpm}$ for $6 \mathrm{~min}$. The cell pellet was resuspended gently in hypotonic solution $(0.4 \% \mathrm{KCl}: 0.4 \%$ sodium citrate) to a final volume of $4 \mathrm{~mL}$. The cell suspension was incubated at $37^{\circ} \mathrm{C}$ for $7 \mathrm{~min}$ to swell the cells. This suspension was then centrifuged at $1000 \mathrm{rpm}$ for $6 \mathrm{~min}$. The supernatant was then carefully removed and the remaining cell pellet was fixed in $2 \mathrm{~mL}$ of fresh fixative $(3: 1 \mathrm{v} / \mathrm{v}$ methanol and acetic acid) at room temperature for $30 \mathrm{~min}$. Fixed cells were then centrifuged at $1000 \mathrm{rpm}$ for $6 \mathrm{~min}$, and the fixing procedure was repeated three times. Following the last centrifugation, cell pellets were resuspended in $1 \mathrm{~mL}$ fixative. A portion of this cell suspension was then placed on a glass slide and stained with Giemsa for $\sim 15$ min. More than 100 chromosomes were observed, and the aberrant chromosomes (e.g., visible breaks, dicentric chromosomes, fragmentations, chromosome loss, ring formation, triradial chromosomes, and gaps) were counted. This data was then used to determine the ratio of aberrant chromosomes. 


\section{Immunofluorescence}

Cells were grown on chamber slides, fixed with $4 \%$ paraformaldehyde, and permeabilized with PBS containing $0.1 \%$ Triton X-100. After blocking with $3 \%$ bovine serum albumin for $1 \mathrm{~h}$, cells were incubated with primary antibodies overnight. These cells were then washed three times with PBS, and incubated with Alexa Fluor 488 goat anti-mouse $\operatorname{IgG}(\mathrm{H}+\mathrm{L})$ or Alexa Fluor 594 goat anti-rabbit IgG $(\mathrm{H}+\mathrm{L})$ secondary antibodies. Nuclei were visualized with DAPI staining. Fluorescence signals were analyzed using an Olympus Fluorview 1000 confocal microscope.

\section{Animal studies}

Athymic BALB/c 4-6 weeks old nude mice, purchased from Beijing HFK Bioscience Co., Ltd (Beijing, China), were housed in a specific pathogen-free room with a $12 \mathrm{~h}$ light/dark cycle at $25 \pm 1^{\circ} \mathrm{C}$ and fed an autoclaved chow diet and water ad libitum. All experiments were performed according to the institutional ethical guidelines on animal care and approved by the Institute Animal Care and Use Committee at Shanghai Institute of Materia Medica. HT29 and SW620 cells were s.c. injected into the right flank of nude mice at $5 \times 10^{6}$ cells/mouse (six mice per group). Tumor diameters were measured two times per week and tumor volumes $(\mathrm{V})$ calculated using $1 / 2 \times$ length $\times$ width $^{2}$.

\section{ACKNOWLEDGEMENT}

We thank Professor Jian Jin (University of North Carolina at Chapel Hill) for the gift of the plasmids pLKO.1-shControl and pLKO.1-shG9a. We are grateful to Martin J. Walsh for the plasmids pEGFP-hG9a and pEGFP- $\triangle$ SET-hG9a. This work was supported in part by grants from the National Basic Research Program of China (2013CB932503) and the National Natural Science Foundation of China $(81273545,81321092)$.

\section{REFERENCES}

1. Weitz J, Koch M, Debus J, Höhler T, Galle PR, Büchler MW. Colorectal cancer. The Lancet. 2005; 365:153-165.

2. Ross W, Lynch P, Raju G, Rodriguez A, Burke T, Hafemeister L, Hawk E, Wu X, Dubois RN, Mishra L. Biomarkers, bundled payments, and colorectal cancer care. Genes Cancer. 2012; 3:16-22.

3. WHO GLOBOCAN 2008: 2008; http://www.iarc.fr/en/ publications/pdfs-online/wcr/2003/index.php.

4. Stewart BW, Kleihues P. World Cancer Report. IARCPress. Lyon: 2003; page 1-351.
5. Rodríguez-Moranta F, Saló J, Arcusa A, Boadas J, Piñol V, Bessa X, Batiste-Alentorn E, Lacy AM, Delgado S, Maurel J, Piqué JM, Castells A. Postoperactive surveillance in patients with colorectal cancer who have undergone curative resection: A prospective, multicenter, randomized, controlled trial. J. Clin. Oncol. 2006; 24:386-393.

6. Jemal A, Bray F, Center MM, Ferlay J, Ward E, Foman D. Global cancer statistics. CA Cancer J Clin. 2011; 61:69-90.

7. Mundade RIT, Prabhu L, Loehrer PJ, Lu T. Genetic pathways, prevention, and treatment of sporadic colorectal cancer. Oncoscience. 2014; 1:400-406.

8. Shao RG, Cao CX, Zhang H, Kohn KW, Wold MS, Pommier Y. Replication-mediated DNA damage by camptothecin induces phosphorylation of RPA by DNAdependent protein kinase and dissociates RPA:DNA-PK complexes. The EMBO Journal. 1999; 18:1397-1406.

9. Vogelstein B, Papadopoulos N, Velculescu VE, Zhou S, Diaz LA Jr, Kinzler KW. Cancer genome landscapes. Science. 2013; 339:1546-1558.

10. Tachibana M, Sugimoto K, Nozaki M, Ueda J, Ohta T, Ohki M, Fukuda M, Takeda N, Niida H, Kato H, Shinkai Y. G9a histone methyltransferase plays a dominant role in euchromatic histone $\mathrm{H} 3$ lysine 9 methylation and is essential for early embryogenesis. Genes Dev. 2002; 16:1779-1791.

11. Huang J, Dorsey J, Chuikov S, Perez-Burgos L, Zhang X, Jenuwein T, Reinberg D, Berger SL. G9a and Glp methylate lysine 373 in the tumor suppressor p53. J. Biol. Chem. 2010; 285:9636-9641.

12. Schaefer A, Sampath SC, Intrator A, Min A, Gertler TS, Surmeier DJ, Tarakhovsky A, Greengard P. Control of cognition and adaptive behavior by the GLP/G9a epigenetic suppressor complex. Neuron. 2009; 64:678-691.

13. Imai $\mathrm{K}$, Togami $\mathrm{H}$, Okamoto $\mathrm{T}$. Involvement of histone $\mathrm{H} 3$ Lysine 9 (H3K9) methyl transferase G9a in the maintenance of HIV-1 latency and its reactivation by BIX01294. J. Biol. Chem. 2010; 285:16538-16545.

14. Kondo Y, Shen L, Issa JP. Critical role of histone methylation in tumor srppressor gene silencing in colorectal cancer. Mol Cell Biol. 2003; 23:206-215.

15. Kondo Y, Shen L, Ahmed S, Boumber Y, Sekido Y, Haddad BR, Issa JP. Downregulation of histone H3 lysine 9 methyltransferase G9a induces centrosome disruption and chromosome instability in cancer cells. PLoS One. 2008; 3:e2037.

16. Marder BA, Morgan WF. Delayed chromosomal instability induced by DNA damage. Mol Cell Biol. 1993; 13:6667-6677.

17. Collins AR. The comet assay for DNA damage and repair: principles, applications, and limitations. Mol. Biotechnol. 2004; 26:249-261.

18. Srivastava N, Gochhait S, de Boer P, Bamezai RN. Role of $\mathrm{H} 2 \mathrm{AX}$ in DNA damage response and human cancers. Mutat Res. 2009; 681:180-188. 
19. Taylor RC, Cullen SP, Martin SJ. Apoptosis: controlled demolition at the cellular level. Nat Rev Mol Cell Biol. 2008; 9:231-241.

20. d'Adda di Fagagna F. Living on a break: cellular senescence as a DNA-damage response. Nat Rev Cancer. 2008; 8:512-522.

21. Pospelova, Demidenko ZN, Bukreeva EI, Pospelov VA, Gudkov AV, Blagosklonny MV. Pseudo-DNA damage response in senescent cells. Cell cycle. 2009; 8:4112-4118.

22. Leontieva OV, Lenzo F, Demidenko ZN, Blagosklonny MV. Hyper-mitogenic drive coexists with mitotic incompetence in senescent cells. Cell cycle. 2012; 11:4642-4649.

23. Dimri GP, Lee $X$, Basile G, Acosta M, Scott G, Roskelley C, Medrano EE, Linskens M, Rubelj I, PereiraSmith O, Peacocke M, Campisi J. A biomarker that identifies senescent human cells in culture and in aging skin in vivo. PNAS. 1995; 92:9363-9367.

24. Chou TC, Talalay P. Analysis of combined drug effects: a new look at a very old problem. Trends Pharmacol. Sci. $1983 ; 4: 450-454$.

25. Chen MW, Hua KT, Kao HJ, Chi CC, Wei LH, Johansson G, Shiah SG, Chen PS, Jeng YM, Cheng TY, Lai TC, Chang JS, Jan YH, et al. H3K9 histone methyltransferase G9a promotes lung cancer invasion and metastasis by silencing the cell adhesion molecule Ep-CAM. Cancer Res. 2010; 70:7830-7840.

26. Wang L, Xu S, Lee JE, Baldridge A, Grullon S, Peng W, Ge K. Histone H3K9 methyltransferase G9a represses PPARgamma expression and adipogenesis. EMBO J. 2013; $32: 45-59$.

27. Kim JT, Li J, Jang ER, Gulhati P, Rychahou PG, Napier DL, Wang C, Weiss HL, Lee EY, Anthony L, Townsend CM Jr, Liu C, Evers BM. Deregulation of Wnt/ beta-catenin signaling through genetic or epigenetic alterations in human neuroendocrine tumors. Carcinogenesis. 2013; 34:953-961.

28. Fnu S, Williamson EA, De Haro LP, Brenneman M, Wray J, Shaheen M, Radhakrishnan K, Lee SH,
Nickoloff JA, Hromas R. Methylation of histone H3 lysine 36 enhances DNA repair by nonhomologous end-joining. PNAS. 2011; 108:540-545.

29. Nguyen AT, Zhang Y. The diverse functions of Dot1 and H3K79 methylation. Genes Dev. 2011; 25:1345-1358.

30. FitzGerald J, Moureau S, Drogaris P, O'Connell E, Abshiru N, Verreault A, Thibault P, Grenon M, Lowndes NF. Regulation of the DNA damage response and gene expression by the Dot1L histone methyltransferase and the 53Bp1 tumour suppressor. PLoS One. 2011; 6:e14714.

31. Wakeman TP, Wang Q, Feng J, Wang XF. Bat3 facilitates H3K79 dimethylation by DOT1L and promotes DNA damage-induced 53BP1 foci at G1/G2 cell-cycle phases. EMBO J. 2012; 31:2169-2181.

32. Wu Z, Lee ST, Qiao Y, Li Z, Lee PL, Lee YJ, Jiang X, Tan J, Aau M, Lim CZ, Yu Q. Polycomb protein EZH2 regulates cancer cell fate decision in response to DNA damage. Cell Death Differ. 2011; 18:1771-1779.

33. Tachibana M, Sugimoto K, Nozaki M, Ueda J, Ohta T, Ohki M, Fukuda M, Takeda N, Niida H, Kato H, Shinkai Y. G9a histone methyltransferase plays a dominant role in euchromatic histone H3 lysine 9 methylation and is essential for early embryogenesis. Genes Dev. 2002; 16:1779-1791.

34. Juergens RA, Wrangle J, Vendetti FP, Murphy SC, Zhao M, Coleman B, Sebree R, Rodgers K, Hooker CM, Franco N, Lee B, Tsai S, Delgado IE, et al. Combination Epigenetic Therapy Has Efficacy in Patients with Refractory Advanced Non-Small Cell Lung Cancer. Cancer Discov. 2011; 1:598-607.

35. Zhu XD, Zhang JB, Zhuang PY, Zhu HG, Zhang W, Xiong YQ, Wu WZ, Wang L, Tang ZY, Sun HC. High expression of macrophage colony-stimulating factor in peritumoral liver tissue is associated with poor survival after curative resection of hepatocellular carcinoma. J. Clin. Oncol. 2008; 26:2707-2716.

36. Nishio H, Walsh MJ. CCAAT displacement proteincut homolog recruits G9a histone lysine methyltransferase to repress transcription. PNAS. 2004; 101:11257-11262. 


\section{SUPPLEMENTARY FIGURES}

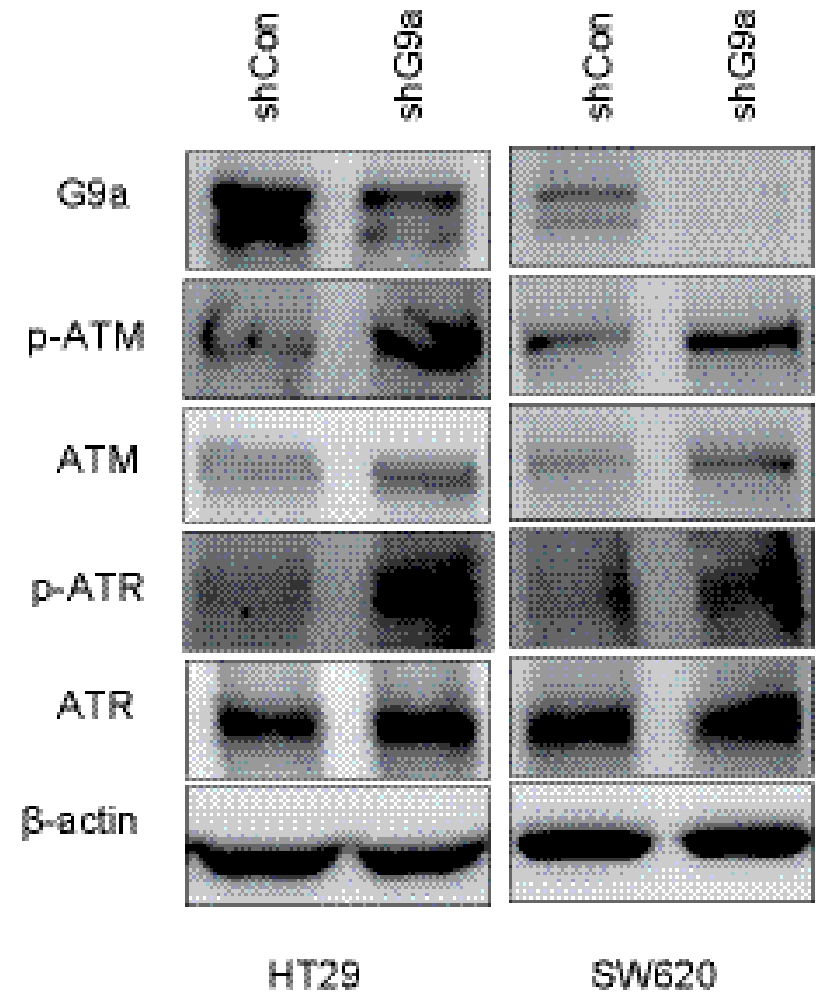

Supplementary Figure S1: Depletion of G9a upregulates the protein level of p-ATM and p-ATR. Protein levels of G9a, p-ATM, ATM, p-ATR, ATR and $\beta$-actin (loading control) from HT29 and SW620 cells that stably suppress G9a. Each condition was studied at $n \geq 3$.

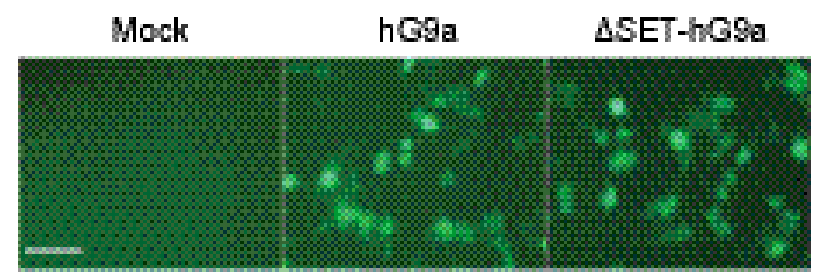

2937

Supplementary Figure S2: The representative pictures of 293T cell after stable transfection with GFP-taged full length or SET domain deleted G9a (scale bar $25 \mu \mathrm{M})$. 


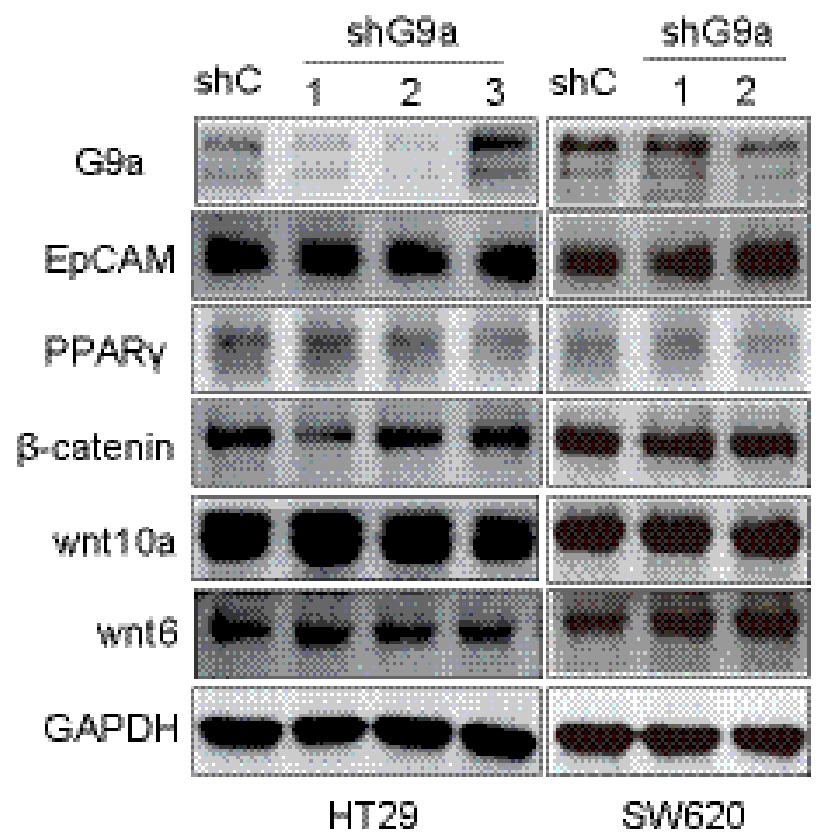

Supplementary Figure S3: Depletion of G9a has no effect on EpCAM, PPAR $\gamma$, and Wnt expression. Protein levels of G9a, EpCAM, PPAR $\gamma, \beta$-catenin, Wnt6, Wnt10a and GAPDH (loading control) from HT29 and SW620 cells that stably suppress G9a. Each condition was studied at $n \geq 3$. 